$\underline{\text { Snippets }}$

\title{
Snippets from the world wide web
}

\author{
Sri Lanka Journal of Child Health, 2007; 36: 22-23
}

Sedentary Lifestyle, BMI Linked to Blood

Pressure in US Adolescents

In teens with sedentary lifestyles, each increase in sedentary activity by 1 hour was linked with an increase in systolic blood pressure.

http://mp.medscape.com/cgibin1/DM/y/eBJA60EIZ1O0DzQ0ILto0Eb

\section{MMR Vaccination May Be Safe, Effective in Juvenile Idiopathic Arthritis}

In a retrospective study, the measles, mumps, rubella booster vaccination was safe and effective in children with juvenile idiopathic arthritis; disease activity was not affected by the vaccine.

http://mp.medscape.com/cgibin1/DM/y/eBJA60EIZ1O0DzQ0ILv70Eh

Second Dose of Measles-Mumps-Rubella-Varicella Vaccine Safe and Immunogenic

An experimental measles-mumps-rubella-varicella (MMRV) vaccine when given as a second dose is safe and immunogenic in healthy children aged 5 to 6 years, according to a report in the February issue of The Pediatric Infectious Disease Journal.

http://mp.medscape.com/cgi-

bin1/DM/y/eBJA60EIZ1O0DzQ0ILv60Eg

\section{WHO Backs Glaxo's Rotarix for Mass Vaccination}

GlaxoSmithKline Plc's vaccine Rotarix, designed to prevent gastroenteritis caused by rotavirus in children, has been endorsed as suitable for use by U.N. agencies by the World Health Organization.

http://mp.medscape.com/cgibin1/DM/y/eBJA60EIZ1O0DzQ0ILvS0EB

\section{Vaccination Status Has No Effect on Eczema or Wheeze in Infants}

Whether children have been vaccinated against diphtheria, pertussis, poliomyelitis, tetanus, and Haemophilus influenzae type $b$ in the first sixmonths of life seems to have little effect on the incidence of eczema or recurrent wheeze at 1 year, Dutch researchers report.

http://mp.medscape.com/cgibin1/DM/y/eBJA60EIZ1O0DzQ0ILtW0ED

\section{Perineal Sensation in Open Spina Bifida Predicts Outcome}

In infants with open spina bifida, the presence of perineal sensation appears to be associated with significantly better long-term outcome, according to UK researchers.

http://mp.medscape.com/cgibin1/DM/y/eBJA60EIZ1O0DzQ0ILWr0EB

\section{Simple Criteria Predict Low Risk of Serious Bacterial Infections in Febrile Neonates}

A simple set of criteria can be used in place of a full investigation for sepsis to determine the likelihood of serious bacterial infections (SBI) in febrile neonates, according to a study published in the January issue of the Archives of Diseases in Childhood, Fetal and Neonatal Edition.

http://mp.medscape.com/cgibin1/DM/y/eBJA60EIZ1O0DzQ0ILwo0Ee

Repeat Corticosteroids to Speed Fetal Lung Development Should Be Limited

Multiple courses in women with high-risk preterm birth suggest risk for cerebral palsy in infant.

http://mp.medscape.com/cgibin1/DM/y/eBJA60EIZ1O0DzQ0ILu50Ee

\section{Beta-Blockade Limits Aortic Dilatation in Children With Marfan Syndrome}

Aortic dilatation during childhood in patients with Marfan syndrome can be reduced by beta-blocker therapy, according to a report in the February 1st American Journal of Cardiology. 
http://mp.medscape.com/cgi-

bin1/DM/y/eBJA60EIZ1O0DzQ0ILtx0Ek

\section{Corticosteroids of No Benefit in Kawasaki Disease}

A new study trial has failed to show any benefit of the corticosteroid methylprednisolone when added to usual treatment for acute Kawasaki disease, an acute vasculitis of unknown cause that can have coronary sequelae.

http://mp.medscape.com/cgi-

bin1/DM/y/eBJA60EIZ1O0DzQ0ILty0El

\section{Low-Cost Bottle-Spacer Bronchodilator Treatment May Be Effective in Children}

In children with acute obstruction of the lower airway, a low-cost bottle-spacer was as effective as a conventional spacer for bronchodilator treatment.

http://mp.medscape.com/cgibin1/DM/y/eBIgW0EIZ1O0DzQ0IJtv0Eq

\section{Maternal Alcohol Consumption During Pregnancy Increases Risk for Cryptorchidism}

In a Danish-Finnish study, regular alcohol intake during pregnancy appeared to increase the risk for congenital cryptorchidism in boys; the mechanisms for this association are unknown.

http://mp.medscape.com/cgibin1/DM/y/eBIgW0EIZ1O0DzQ0IKch0EM

\section{Pneumococcal Vaccination of Infants Cost- Effective in Developing Countries}

Routine pneumococcal vaccination of infants in developing countries is estimated to be highly costeffective and associated with a marked reduction in childhood mortality, according to a study in the February 3rd issue of The Lancet.

http://mp.medscape.com/cgibin1/DM/y/eBIgW0EIZ1O0DzQ0IKbH0En

\section{Breast-Feeding Enhances Vision Development in Infants}

Breast-fed children are significantly more likely to do well in measures of foveal stereoacuity than are those who received formula, according to UK researchers. http://mp.medscape.com/cgibin1/DM/y/eBIgW0EIZ1O0DzQ0IKcw0Eb

\section{Apolipoprotein E Genotypes Increase Risk of Cerebral Palsy}

Children who carry the apolipoprotein E (APOE) e4 or e2 genotypes are increased risk for developing cerebral palsy (CP), new research suggests. Moreover, carriage of the e4 allele seems to increase the severity of disease and may predispose to microcephaly.

http://mp.medscape.com/cgibin1/DM/y/eBIgW0EIZ1O0DzQ0IJyV0EP

\section{Montelukast Reduces Bronchial Hyperreactivity in Asthmatic Children}

The results of a study published in the January issue of Chest suggest that 4 weeks of oral montelukast treatment decreases bronchial hyperreactivity in preschool children with asthma.

http://mp.medscape.com/cgibin1/DM/y/eBIgW0EIZ1O0DzQ0IJtx0Es

Prenatal Folic Acid Reduces Risk for Isolated Cleft Lip

A study showed that folic acid supplements during early pregnancy were effective in reducing the risk for isolated cleft lip in infants by about one third.

http://mp.medscape.com/cgibin1/DM/y/eBIDG0EIZ1O0DzQ0IJGC0EP

\section{Decline Seen in the Prevalence of Penicillin Sensitization}

Results of a study published in the December issue of the Annals of Allergy, Asthma, and Immunology indicate that there has been a marked decline in the prevalence of penicillin sensitization in the pediatric population over a 10 -year period.

http://mp.medscape.com/cgibin1/DM/y/eBIDG0EIZ1O0DzQ0IJEg0Ex

\section{B J C Perera \\ Joint Editor}

